\title{
EVALUACIÓN DEL CARBONO ACUMULADO EN SUELO EN SISTEMAS SILVOPASTORILES DEL CARIBE COLOMBIANO*
}

\author{
José Luis Contreras-Santos ${ }^{1 / * *}$, Judith Martínez-Atencia ${ }^{2}$, Jorge Cadena-Torres $^{3}$, \\ Cindy Katherine Falla-Guzmán ${ }^{4}$
}

Palabras clave: Ganadería; pasturas; sistemas silvopastoriles; carbono orgánico en el suelo.

Keywords: Llivestock; pastures; silvopastoral systems; organic carbon in the soil.

Recibido: 08/10/18

RESUMEN

Los sistemas silvopastoriles juegan un papel importante en la recuperación de suelos degradados y en la mitigación de los gases de efecto invernadero, debido a su capacidad para capturar el carbono atmosférico e inmovilizarlo en el suelo. Para estimar el potencial de los sistemas silvopastoriles para incorporar carbono al suelo, se evaluaron 4 sistemas de diferente grado de complejidad estructural, frente a una pastura sin árboles, en un sistema ganadero del Caribe Colombiano. Las evaluaciones incluyeron mediciones de la cantidad de carbono orgánico acumulado, las propiedades físicas del suelo como densidad aparente y resistencia mecánica a la penetración, y la biomasa de raíces finas. Los resultados mostraron que en promedio los arreglos silvopastoriles presentaron una acumulación de carbono que varió entre 60,6 y 65,1 t.ha ${ }^{-1}$ de $C$, en comparación con el sistema tradicional ganadero, de pasturas sin árboles, en el cual se detectó una acumulación de 38,3 t.ha ${ }^{-1}$

\footnotetext{
* Proyecto financiado por el Ministerio de Agricultura y Desarrollo Rural y la Corporación Colombiana de Investigación Agropecuaria (Agrosavia).

** Autor para correspondencia. Correo electrónico: jlcontreras@agrosavia.co

1 Corporación Colombiana de Investigación Agropecuaria (Agrosavia), Centro de Investigaciones Turipaná, Colombia. (iD) 0000-0002-8179-3430.

2 Corporación Colombiana de Investigación Agropecuaria (Agrosavia), Centro de Investigaciones Turipaná, Colombia. (iD) 0000-0002-8275-2956.
}

Aceptado: 07/03/19

\section{ABSTRACT}

Evaluation of carbon accumulated in soil in silvopastoral systems of the Colombian Caribbean. The silvopastoral systems play an important role in the recovery of degraded soils and in the mitigation of greenhouse gases, due to their capacity to capture atmospheric carbon and immobilize it in the soil. To estimate the potential of silvopastoral systems to incorporate carbon into the soil, 4 silvopastoral systems with different degrees of structural complexity were evaluated against a treeless pasture in a Colombian Caribbean livestock system. The evaluations included measurements of the amount of organic carbon accumulated, the physical properties of the soil as apparent density and mechanical resistance to penetration, and the biomass of fine roots. The results showed that on average the silvopastoral arrangements showed a carbon accumulation that varied between 60.6 and $65.1 \mathrm{t}^{-h a^{-1}}$ of $\mathrm{C}$, in comparison with the traditional livestock system, of treeless pastures,

3 Corporación Colombiana de Investigación Agropecuaria (Agrosavia), Centro de Investigaciones Turipaná, Colombia. (iD) 0000-0002-5180-2893.

4 Corporación Colombiana de Investigación Agropecuaria (Agrosavia), Centro de Investigaciones Turipaná, Colombia. (iD) 0000-0002-1266-8505. 
de C. Lo anterior, representa una acumulación de carbono que es entre 58,2 y $69,9 \%$ superior en los sistemas silvopastoriles, comparada con el sistema tradicional de producción ganadera, basada en solo pasturas. Igualmente, se observó una reducción en la densidad aparente del suelo y la resistencia a la penetración de las raíces, concomitantes con una mayor presencia de raíces finas en los sistemas silvopastoriles, en comparación con lo observado en el suelo de la pastura sin árboles. Estos resultados demuestran los efectos benéficos de los sistemas silvopastoriles en la incorporación del carbono al suelo y representan una alternativa viable para el establecimiento de una ganadería más sostenible en el Caribe Colombiano.

\section{INTRODUCCIÓN}

En Colombia hay reportadas alrededor de 34 millones de hectáreas dedicadas a la ganadería, lo que equivale a aproximadamente el 30\% del suelo disponible para sistemas productivos en el país. Bajo este sistema de producción operan modelos de ganadería de tipo extensiva con muy baja carga animal y en donde la principal fuente de alimentación del animal es la pastura. Este sistema de explotación ha conllevado al remplazo de áreas boscosas para establecer zonas de pastoreo de ganado (Mora et al. 2017).

En Colombia, el continuo uso de las áreas para la explotación ganadera ha ocasionado que aproximadamente el $77 \%$ de los suelos en zonas ganaderas presenten algún grado de degradación, la cual ha sido calificada de moderada a severa (Murgueitio et al. 2011). Se reconoce que las principales causas de esta degradación son la deforestación, cambio de uso del suelo y la compactación del suelo, debida al pisoteo continuo de los animales (Mora et al. 2017, IDEAM y UDCA 2015, Ortega 2007). in which an accumulation of 38.3 t.ha ${ }^{-1}$ of $\mathrm{C}$. This represents an accumulation of carbon that is between 58.2 and $69.9 \%$ higher in silvopastoral systems, compared with the traditional system of livestock production, based on only pastures. Likewise, a reduction in the apparent density of the soil and resistance to root penetration was observed, concomitant with a greater presence of fine roots in the silvopastoral systems, in comparison with what was observed in the soil of the treeless pasture. These results demonstrate the beneficial effects of silvopastoral systems on the incorporation of carbon into the soil and represent a viable alternative for the establishment of a more sustainable livestock in the Colombian Caribbean.

Como alternativas integradoras de procesos sostenibles, productivos, viables y alineados con servicios ecosistémicos, se ha planteado el establecimiento de sistemas silvopastoriles, que contribuyen a la recuperación y rehabilitación de la capacidad productiva del suelo. En estos sistemas silvopastoriles se integran una variedad de especies vegetales, entre ellas gramíneas, especies arbustivas y arbóreas, las cuales son capaces de aportar alimentos y bienestar para el ganado, pero, además, generan aportes significativos de materia orgánica al suelo, a través de la acumulación de hojarasca, tallos y producción de biomasa de raíces finas (Ramachandran et al. 2009, Murgueitio et al. 2011, Vallejo et al. 2012, Martínez et al. 2014). Sin embargo, en la Región Caribe en Colombia, estos sistemas han sido poco implementados. Una de las principales causas de su baja adopción son los costos asociados a su establecimiento, pero también al desconocimiento de las ventajas que este tipo de sistemas tendrían para rehabilitar y recuperar suelos deteriorados (Ledesma 2003).

Diferentes estudios han demostrado que el establecimiento de sistemas silvopastoriles 
disminuye la compactación del suelo, aumenta la macro y meso porosidad, mejora la dinámica de nutrientes y la conservación del agua en el suelo fomenta la actividad microbial, entre muchos otros beneficios (Vallejo et al. 2012, Montagnini et al. 2015). Igualmente, se reconoce que estos sistemas son importantes sumideros de carbono en el suelo, sin embargo, en la región Caribe de Colombia son pocos los estudios que han cuantificado o valorado sus aportes (Rojas et al. 2009). El presente estudio se llevó a cabo con el objeto de cuantificar la cantidad de carbono acumulado en el suelo bajo sistemas silvopastoriles en la región Caribe de Colombia y medir sus efectos sobre algunas propiedades físicas del suelo.

\section{MATERIALES Y MÉTODOS}

\section{Localización}

El estudio se llevó a cabo en las instalaciones del Centro de Investigaciones Turipaná de propiedad de la Corporación Colombiana de Investigación Agropecuaria (Agrosavia), localizado en el municipio de Cereté, departamento de Córdoba, en Colombia, ubicado a $75^{\circ} 47^{\prime}$ de longitud Oeste y $8^{\circ} 51^{\prime}$ de latitud Norte, a $15 \mathrm{msnm}$.
Este centro presenta una temperatura media anual $28^{\circ} \mathrm{C}$, precipitación $1200 \mathrm{~mm}$ y humedad relativa del $82 \%$ (Barragan et al. 2015). La precipitación presenta un comportamiento bimodal, con un periodo lluvioso, comprendido entre abril y noviembre, seguido de un periodo seco, comprendido entre diciembre y marzo (Barragán et al. 2015). De acuerdo con Holdridge (2000), el área de estudio pertenece a la zona agroecológica Bosque Seco Tropical (BsT).

\section{Tratamientos}

El experimento se realizó en un sistema silvopastoril establecido en 1998 por Cajas y Sinclair (2001), en el Centro de Investigaciones Turipaná, el cual ha servido como modelo para la realización de diversos tipos de investigaciones que han sido ampliamente reportadas en la literatura (Barragán et al. 2015, Suarez 2014, Linares 2006, Ayazo y Soto 2007). Bajo este sistema se encuentran establecidos diferentes arreglos que combinan especies arbóreas y arbustivas con capacidad para suministrar alimentación a los animales, junto a la pastura. En este experimento se consideró la evaluación de 5 tratamientos consistentes en los arreglos que resultan de combinar las diferentes especies arbóreas y arbustivas, como se muestran en la Tabla 1.

Tabla 1. Tratamientos empleados para la evaluación del carbono inmovilizado en suelo bajo diferentes arreglos silvopastoriles.

\begin{tabular}{lll}
\hline N $^{\text {o }}$ & Tratamiento & Sistema silvopastoril \\
\hline 1 & PS & Pastura sin arboles (testigo modal) \\
2 & SSP1 & Pastura + arbustos forrajeros \\
3 & SSP2 & Pastura + arboles forrajeros \\
4 & SSP3 & Pastura + arbustos forrajeros + arboles forrajeros \\
5 & SSP4 & Pastura + arbustos forrajeros + arboles forrajeros + arboles maderables \\
\hline
\end{tabular}

La pastura establecida en los sistemas silvopastoriles correspondió al pasto Guinea (Panicum maximum) cv Mombasa; las especies arbustivas incluidas fueron leucaena (Leucaena leucocephala) $y$ totumo (Crescentia cujete). Las especies arbóreas forrajeras fueron guácimo (Guazuma ulmifolia), cañafístula (Cassia fistula) y samán (Albizia saman). Los árboles maderables incluidos en el quinto tratamiento fueron ceiba tolúa (Pachira quinata) y caoba (Swietenia macrophylla). 
Estos sistemas se encuentran en un arreglo de $16 \mathrm{~m}$ x $16 \mathrm{~m}$, se intercambiaron las especies arbustivas y arbóreas, lo cual equivale a una densidad de 39 árboles.ha ${ }^{-1}$. Cada tratamiento tenía unas dimensiones de $200 \mathrm{~m}$ de largo por $100 \mathrm{~m}$ de ancho, para un total de 2 hectáreas por tratamiento. Lo anterior arrojó un área experimental de 30 hectáreas, correspondientes a los 5 tratamientos y 3 repeticiones, tal como lo describe Barragan et al. (2015).

\section{Diseño experimental}

Se utilizó un diseño experimental de bloques completos al azar (BCA) con 5 tratamientos y 3 repeticiones. El factor de bloqueo correspondió a la condición del drenaje natural, clasificado como rápido, moderado y lento. Los tratamientos correspondieron a la comparación de 4 arreglos silvopastoriles, con diferente complejidad estructural, frente a un testigo modal, correspondiente a la pastura sin árboles. La unidad experimental estaba conformada por potreros de 2,0 hectáreas. En la Figura 1 se muestra la distribución de los tratamientos en el área experimental.

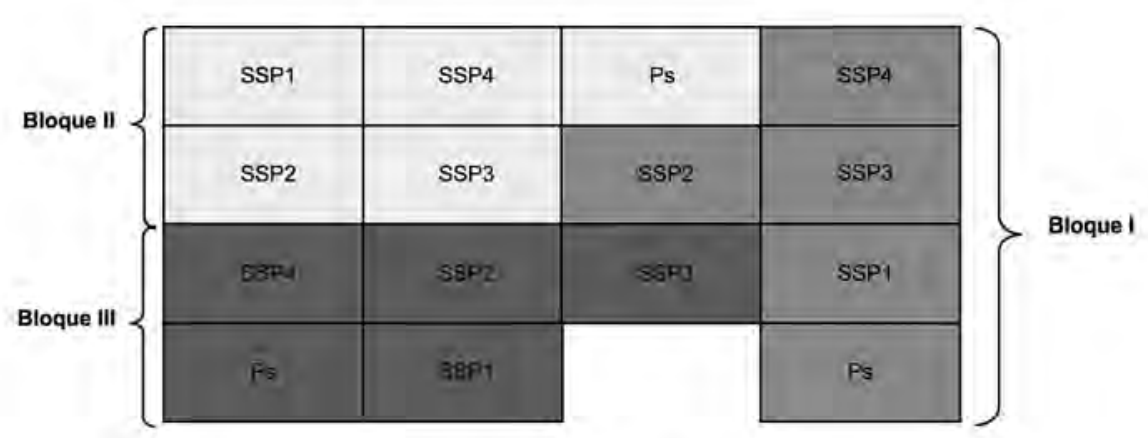

Figura 1 Distribución de los tratamientos de sistemas silvopastoriles dentro del área experimental.

El suelo del área experimental corresponde a un Vertic Endoaquepts (Soil Survey Staff 2014), perteneciente a la serie La Pozona, el cual presenta predomino de textura arcillosas, de tipo expansiva (tipo 2:1), régimen de humedad acuico y temperatura isohipertérmico, con secuencia de horizontes $\mathrm{Ap}, \mathrm{Bg}, \mathrm{Bg} 2$ y $\mathrm{Cg}$. Igualmente, presenta una distribución irregular de carbono orgánico y alto contenido de bases intercambiables.

\section{Variables evaluadas}

En cada tratamiento, se tomaron muestras de suelo en 5 puntos de la zona central de cada parcela y, en cada punto, se tomaron 5 submuestras de suelo en los primeros $20 \mathrm{~cm}$ de profundidad (Figura 2). Las submuestras correspondientes a cada punto de muestreo fueron mezcladas, homogenizadas y enviadas al Laboratorio de Química de Suelos de Agrosavia, en el Centro de Investigaciones Tibaitatá en Bogotá, donde se realizaron análisis de carbono orgánico. En el laboratorio se realizó la determinación de carbono orgánico mediante el método de combustión húmeda descrito por Walkley y Black (1934). 


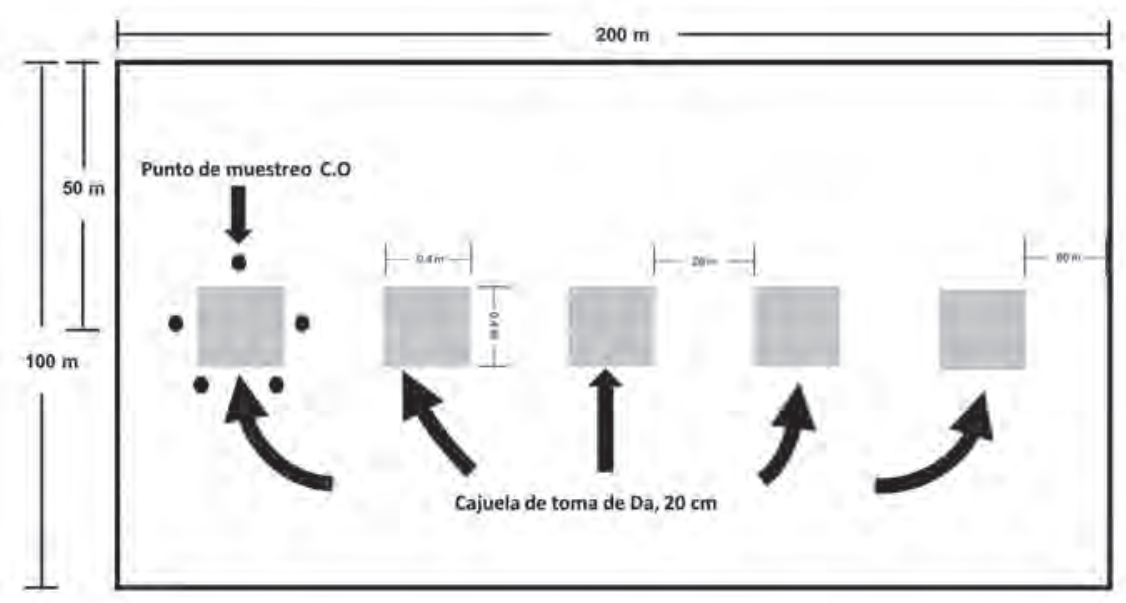

Figura 2 Distribución de puntos de muestreo en cada parcela.

Con los resultados obtenidos en el laboratorio, se determinó la cantidad de carbono orgánico acumulado en el suelo (COS), mediante la siguiente relación propuesta por Rojas et al. (2009):

\section{Carbono orgánico acumulado $(C O S)=C O(\%) \times D a\left(\mathrm{gcm}^{-3}\right) \times \operatorname{Prof}(\mathrm{cm}) \times 100$}

Donde:

$\mathrm{COS}=$ carbono orgánico acumulado en el suelo en t.ha ${ }^{-1}$ de $\mathrm{C}$

CO $(\%)=$ contenido de carbono orgánico en el suelo $(\%)$

$\mathrm{Da}=$ densidad aparente en $\left(\mathrm{g} \mathrm{cm}^{-3}\right)$

Prof. $(\mathrm{cm})=$ profundidad de muestreo

En los mismos 5 puntos de muestreo, se tomaron muestras indisturbadas de suelo a los 10,20 y $30 \mathrm{~cm}$ de profundidad, usando cilindros metálicos, de acuerdo con la metodología descrita por Burt (2014), y estas se usaron para la determinación de la densidad aparente del suelo (Da). Igualmente, se hicieron determinaciones de la resistencia mecánica a la penetración de las raíces (RMP) a las mismas profundidades, mediante la utilización de un Penetrologger Stiboka
(Eijkelkamp). En los mismos sitios de muestreo, se determinó la biomasa de raíces finas $(<5 \mathrm{~mm})$ utilizando un anillo metálico de $10,1 \mathrm{~cm}$ de alto y 9,7 cm de diámetro interno, el cual fue utilizado para tomar una muestra de suelo indisturbado, del que se extrajeron las raíces presentes, según la metodología descrita por Jiménez y Arias (2004). La separación de las raíces de la suspensión de suelo se logró con un elutriador (Fahey et al. 1999), mediante el bombeo de agua a presión a la muestra de suelo contenida en un recipiente cerrado, con un orificio de entrada en la parte inferior y otro de salida en la parte superior. Las raíces finas fueron recogidas en un tamiz (Norma ASTM E - 11/95), número 12 de apertura de $1 \mathrm{~mm}$ (Pire 1985). En laboratorio se determinó la masa de raíces pesando la muestra de raíces finas, secas al horno, a una temperatura de $70^{\circ} \mathrm{C}$ por un periodo de 48 horas (ISO 6496 2016). 


\section{Análisis estadísticos}

Se realizaron análisis de varianza para cada una de las variables de respuesta y en los casos, en los cuales se detectaron diferencias significativas a un nivel de $5 \%$, se realizaron pruebas de separación de medias, mediante la prueba HSD de Tukey. Para lo anterior, se utilizó el programa estadístico SAS (Statistical Analysis Software SAS/STAT, versión 9.4).

\section{RESULTADOS Y DISCUSIÓN}

\section{Carbono orgánico en suelo (COS)}

Los análisis estadísticos mostraron diferencias $(p<0,05)$ entre los diferentes tratamientos, al indicar que existe un efecto de los sistemas silvopastoriles evaluados sobre la cantidad de carbono orgánico acumulado en suelo (COS). Como se muestra en la Figura 3, los mayores valores de carbono orgánico acumulado en suelo se presentaron en los 4 sistemas silvopastoriles (SSP1, SSP2, SSP3 y SSP4), en comparación con el testigo modal de pastura sin árboles (SP).

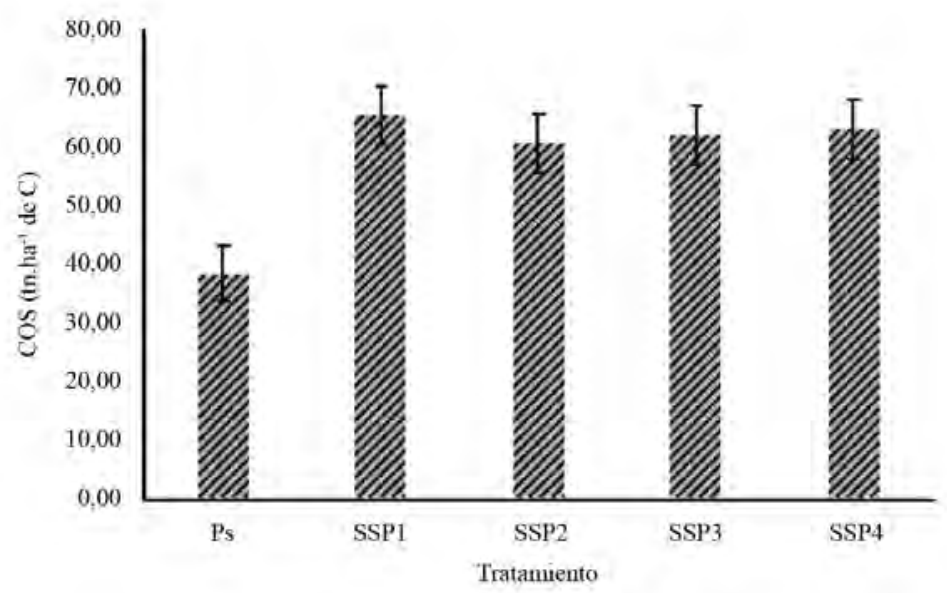

Figura 3 Carbono orgánico acumulado en el suelo (COS) en 4 sistemas silvopastoriles (SSP) en comparación con una pastura sin árboles.

En promedio los arreglos silvopastoriles presentaron una acumulación de carbono de $65,10,60,60,61,80$ y 62,93 t.ha $^{-1}$ de C, en los sistemas silvopastoriles SSP1, SSP2, SSP3 y SSP4, respectivamente, en comparación con el sistema tradicional ganadero, de pasturas sin árboles (PS), en el cual se detectó una acumulación de 38,3 t.ha ${ }^{-1}$ de C. Lo anterior, representa una acumulación de carbono que es en promedio superior entre 58,2 y $69,9 \%$ en los sistemas silvopastoriles, comparada con el sistema tradicional de producción ganadera, basada en solo pasturas. Esto probablemente se debe a la mayor complejidad y diversidad arbórea que representan los sistemas silvopastoriles, los cuales, en su conjunto, al actuar sobre la misma área de terreno, contribuyen en mejor forma con la fijación del carbono atmosférico y su incorporación a estructuras complejas que posteriormente se incorporan al suelo. Los anteriores resultados demuestran la importancia de la implementación de los sistemas silvopastoriles para la captura e inmovilización de carbono, debido a la inclusión de especies arbustivas y arbóreas 
capaces de aportar cantidades apreciables de materia orgánica al suelo. En previos reportes, Stefano y Jacobson (2017) y Priano et al. (2017) reportan que los sistemas, que asocian diferente tipo de especies arbóreas en una misma unidad de terreno, poseen mayor capacidad de acumulación de carbono en el suelo que un sistema sin árboles basado solo en las pasturas. Dollinger y Shibu (2018) y Cáceres (2012), Martínez et al. (2014) y Lal (2011), por su parte, en experimentos similares llevados a cabo en sistemas silvopastoriles, corroboran los anteriores resultados. Rojas et al. (2009) reportó una acumulación de carbono en el suelo bajo sistemas silvopastoriles de $P$. saman y $D$. robinioides asociados con la pastura $B$. brizantha, equivalente a 121,7 y 121,2 t.ha ${ }^{-1}$ de $\mathrm{C}$, respectivamente, en los primeros $20 \mathrm{~cm}$ de profundidad del suelo. Ibrahim et al. (2007), al evaluar suelos bajo distintos usos en Colombia, Costa Rica y Nicaragua, encontraron que las pasturas degradadas no aportan significativamente al secuestro de carbono, mientras que las pasturas mejoradas, combinadas con árboles y los sistemas silvopastoriles, presentan mayores potencialidades de secuestro de carbono, por lo que recomiendan el establecimiento de pasturas mejoradas con alta densidad de árboles.

De igual forma, en estos experimentos no se presentaron diferencias estadísticas en la cantidad de carbono acumulado en el suelo, entre los 4 sistemas silvopastoriles evaluados (SSP1, SSP2, SSP3 y SSP4), lo cual muestra que independientemente de su complejidad, todos los sistemas presentaron igual capacidad de acumulación de carbono en el suelo. En promedio en los suelos de los sistemas silvopastoriles se detectaron acumulaciones de 65,1, 60,6, 61,8 y 62,9 t.ha ${ }^{-1}$ de C en los sistemas SSP1, SSP2, SSP3 y SSP4, sin diferencias estadísticas entre ellos. Se esperaba que, a mayor complejidad del sistema, mayor sería la capacidad de acumulación de carbono, debido a la mayor cantidad de material vegetal aportado al suelo. Sin embargo, en estos resultados se observó que la inclusión de un mayor número de especies en los sistemas no fue suficiente para representar una mayor cantidad de carbono acumulado en el suelo.

\section{Densidad aparente (Da)}

Los resultados, en este caso, mostraron efectos de los tratamientos silvopastoriles sobre la densidad aparente del suelo, pero los efectos solo fueron significativos $(p<0,05)$ a $\operatorname{los} 20$ y $30 \mathrm{~cm}$ de profundidad del suelo. A los $10 \mathrm{~cm}$ de profundidad del suelo todos los tratamientos presentaron similares valores de densidad aparente. Para el caso de $l o s 20 \mathrm{~cm}$ de profundidad, se observó una menor densidad aparente en los suelos bajo los sistemas silvopastoriles, en comparación con el testigo modal de pastura sin árboles. Iguales efectos se presentaron en el muestreo realizado a los $30 \mathrm{~cm}$ de profundidad, lo que indica un efecto de mejoramiento de suelos por parte de los sistemas silvopastoriles (Tabla 2). No se presentaron diferencias entre los sistemas silvopastoriles entre sí (SSP1, SSP2, SSP3 y SSP4), lo cual indica que todos fueron igualmente efectivos en el mejoramiento de la condición del suelo. 
Tabla 2. Densidad aparente del suelo a 3 profundidades en 4 sistemas silvopastoriles en comparación con una pastura sin árboles $\left(\mathrm{g} . \mathrm{cm}^{-3}\right)$.

\begin{tabular}{lccc}
\hline \multirow{2}{*}{ Tratamiento } & \multicolumn{3}{c}{ Profundidad (cm) } \\
\hline PS & 10 & 20 & 30 \\
SSP1 & $1,22 \mathrm{a}$ & $1,54 \mathrm{a}$ & $1,54 \mathrm{a}$ \\
SSP2 & $1,12 \mathrm{a}$ & $1,16 \mathrm{~b}$ & $1,15 \mathrm{~b}$ \\
SSP3 & $1,22 \mathrm{a}$ & $1,24 \mathrm{~b}$ & $1,24 \mathrm{~b}$ \\
SSP4 & $1,22 \mathrm{a}$ & $1,21 \mathrm{~b}$ & $1,22 \mathrm{~b}$ \\
Media & $1,24 \mathrm{a}$ & $1,22 \mathrm{~b}$ & $1,24 \mathrm{~b}$ \\
R Square & 1,21 & 1,27 & 1,28 \\
CV\% & 0,25 & 0,81 & 0,87 \\
D. Stand. & 9,11 & 7,13 & 5,67 \\
Significancia & 0,11 & 0,09 & 0,07 \\
\hline
\end{tabular}

Al respecto Burt (2014) ha indicado que densidades aparentes superiores a $1,39 \mathrm{~g} . \mathrm{cm}^{-3}$, en suelos de textura arcillosa, son indicativos de compactación y pueden ocasionar restricciones al crecimiento y desarrollo de las raíces de las plantas. De acuerdo con esto, en los experimentos, el suelo que presenta las mejores condiciones, para el crecimiento de las raíces de las plantas, se presenta en los tratamientos con los diferentes arreglos silvopastoriles y sus efectos se aprecian mejor a profundidades superiores a los $20 \mathrm{~cm}$. Uno de los factores que podría contribuir con esta característica es una mayor abundancia de raíces a estas profundidades, producto de la presencia de arbustos y árboles que generarían una mayor cantidad de materia orgánica y carbono acumulado, lo cual contribuiría a una mejor agregación y estructura del suelo. Consecuentemente, es de esperar también una mejor aireación y capacidad para mantener el crecimiento de raíces de las plantas en los sistemas silvopastoriles, especialmente a las mayores profundidades del suelo.

\section{Resistencia mecánica a la penetración de las raíces (RMP)}

Los resultados en este caso solo mostraron efectos de los sistemas silvopastoriles en las mediciones realizadas a $\operatorname{los} 20 \mathrm{~cm}$ de profundidad del suelo. En este caso, se observó que los suelos bajo estos sistemas presentaron valores inferiores de resistencia mecánica a la penetración de raíces que el testigo modal de pastura sin árboles. Los valores de resistencia mecánica a la penetración de raíces obtenida, en estos experimentos, fueron entre un 26 y un $37 \%$ inferiores en los sistemas silvopastoriles comparado con el testigo modal de pastura sin árboles, lo que indica una mejor condición de los suelos en los sistemas silvopastoriles para el crecimiento de las raíces de las plantas (Tabla 3). 
Tabla 3. Resistencia mecánica a la penetración de raíces a 3 profundidades del suelo en 4 sistemas silvopastoriles en comparación con una pastura sin árboles $(\mathrm{MPa})$.

\begin{tabular}{llll}
\hline Tratamiento & \multicolumn{3}{c}{ Profundidad del suelo (cm) } \\
\hline PS & 10 & 20 & 30 \\
SSP1 & $2,45 \mathrm{a}$ & $3,07 \mathrm{a}$ & $3,45 \mathrm{a}$ \\
SSP2 & $1,65 \mathrm{a}$ & $2,16 \mathrm{~b}$ & $2,76 \mathrm{a}$ \\
SSP3 & $1,47 \mathrm{a}$ & $2,22 \mathrm{ab}$ & $2,48 \mathrm{a}$ \\
SSP4 & $1,53 \mathrm{a}$ & $1,93 \mathrm{~b}$ & $2,93 \mathrm{a}$ \\
Media & $1,59 \mathrm{a}$ & $2,26 \mathrm{ab}$ & $2,83 \mathrm{a}$ \\
R Square & 1,74 & 2,33 & 2,89 \\
CV\% & 0,55 & 0,76 & 0,49 \\
D. Stand. & 26,06 & 13,21 & 17,52 \\
Significancia & 0,45 & 0,31 & 0,51 \\
\hline
\end{tabular}

\section{Biomasa de raíces finas}

Los análisis estadísticos indicaron diferencias en la biomasa de raíces finas presentes en el suelo en los diferentes tratamientos evaluados en estos ensayos $(\mathrm{p}<0,05)$. Los resultados presentaron que en todas las profundidades del suelo evaluadas se presenta una mayor biomasa de raíces finas en los sistemas silvopastoriles, en comparación con el sistema modal de pastura sin árboles (Tabla 4).

Tabla 4. Biomasa de raíces finas a 3 profundidades del suelo en 4 sistemas silvopastoriles en comparación con una pastura sin árboles $\left(\mathrm{kg} \cdot \mathrm{ha}^{-1}\right)$.

\begin{tabular}{llcc}
\hline Tratamiento & \multicolumn{1}{c}{ Profundidad del suelo (cm) } & 30 \\
\hline PS & 10 & 20 & 8,01 \\
SSP1 & $26,82 \mathrm{~b}$ & $15,37 \mathrm{c}$ & 215,018 \\
SSP2 & $556,78 \mathrm{~b}$ & $288,15 \mathrm{bc}$ & 865,514 \\
SSP3 & $845,51 \mathrm{ab}$ & $978,20 \mathrm{ab}$ & 924,845 \\
SSP4 & $1457,33 \mathrm{ab}$ & $1221,05 \mathrm{a}$ & 1469,87 \\
Media & $2154,56 \mathrm{a}$ & $1864,70 \mathrm{a}$ & 696,653 \\
R Square & 1008,20 & 873,495 & 0,91 \\
CV\% & 0,81 & 0,90 & 32,63 \\
D. Stand. & 53,15 & 36,73 & 227,36 \\
Significancia & 535,86 & 320,89 & $* *$ \\
\hline
\end{tabular}


Dentro de los sistemas silvopastoriles, los que presentaron una mayor biomasa de raíces fueron los sistemas que combinaban el mayor número de especies. Entre estas, las pasturas con los árboles forrajeros (SSP2), las pasturas con los arbustos forrajeros y los árboles forrajeros (SSP3) y la pastura con los arbustos forrajeros, los árboles forrajeros y los árboles maderables (SSP4). Esto indica que entre más compleja la combinación de especies dentro de los sistemas silvopastoriles, mayor es la biomasa de raíces presentes en el suelo, lo cual genera así una relación directamente proporcional entre el número de especies y la biomasa de raíces. Lo anterior, guarda concordancia con el carbono acumulado en suelo, el cual en buena parte es generado por la abundancia de raíces finas.

En relación con la variación de la biomasa de raíces en la profundidad del suelo, se encontró que a medida que aumenta la profundidad, la biomasa de raíces finas disminuye en todos los tratamientos. Esta reducción es especialmente significativa en los sistemas modales de producción que presentan la pastura sin árboles, mientras que en los sistemas silvopastoriles, aun a profundidades de 20 y $30 \mathrm{~cm}$ de suelo se observa una abundante cantidad de biomasa de raíces finas. Esto se debe a que la mayor diversidad de especies, que se encuentra en los sistemas silvopastoriles, conlleva a una mayor concentración de raíces, donde también se registran las mejores condiciones para el crecimiento de las raíces, tales como menores valores de densidad aparente y menor resistencia mecánica a la penetración.

En general, los anteriores resultados indican que en la región Caribe de Colombia, el establecimiento de sistemas silvopastoriles es una alternativa ecológicamente viable y necesaria a fin de contrarrestar el grado de deterioro que están sufriendo los suelos dedicados a la explotación ganadera. Según estos resultados, el asocio de especies arbustivas y arbóreas, para el establecimiento de los sistemas silvopastoriles, conducen a una mayor capacidad de incorporación de carbono orgánico en el suelo que un sistema de producción ganadera basado solo en gramíneas sin árboles, y por otro lado, la compactación que es ocasionada por el continuo pisoteo del ganado también puede ser contrarrestada con el establecimiento de sistemas ganaderos más complejos.

Durante el desarrollo de estos experimentos, se mantuvo una carga de 4 animales por hectárea y, como se observa en los resultados obtenidos en estos experimentos, el efecto del pisoteo de los animales sobre el suelo fue menor en los sistemas silviopatoriles que el sistema de pastura sin árboles. Los efectos benéficos de la diversidad arbórea presente en estos sistemas sobre la compactación son más evidentes a los $20 \mathrm{~cm}$ de profundidad del suelo, en donde se observa una menor densidad aparente y menor resistencia a la penetración, lo cual favorece el crecimiento y la presencia de una abundante cantidad de raíces, que es favorecida por la presencia de árboles y arbustos en los sistemas solvopastoriles. Por el contrario, bajo el sistema con pastura sin árboles, el pisoteo de los animales induce una mayor compactación del suelo, el cual presenta valores de densidad aparente, especialmente después de los $20 \mathrm{~cm}$ de profundidad, que son considerados indicativos de restricciones severas para el crecimiento de las raíces de las plantas. $\mathrm{Al}$ respecto, varios autores reportan que valores bajos de compactación, dentro de los sistemas silvopastoriles, se deben a la mejor agregación del suelo producto de la materia orgánica que es acumulada, que de paso facilita la aireación, el movimiento y retención del agua en el suelo, acompañado todo ello con una mejor actividad biológica en el suelo (Vallejo et al. 2012, Camargo et al. 2011). Los análisis de correlación efectuados en estos experimentos mostraron una significativa e inversa correlación entre el carbono orgánico acumulado en suelo y la resistencia mecánica a la penetración de raíces, lo que favorece el crecimiento de raíces, lo cual corrobora los anteriores resultados. En estos experimentos no se encontraron correlaciones significativas en los primeros $10 \mathrm{~cm}$ de profundidad del suelo, entre el carbono orgánico acumulado y la densidad aparente. 
Con respecto a lo mencionado, García et al. (2018), Santana et al. (2012) y Cáceres (2012), reportan resultados similares, al observar que bajo los sistemas silvopastoriles los indicadores de compactación de suelo se reducen, lo que se asocia a altos contenidos de materia orgánica. Stefano y Jacobson (2017) y Delgado et al. (2008) reportan, igualmente, que existe una relación directa y positiva entre el contenido de materia orgánica en el suelo y los valores de compactación de suelo.

\section{CONCLUSIONES}

En los sistemas ganaderos de la región Caribe en Colombia, el asocio de especies vegetales, en el establecimiento de sistemas silvopastoriles, conduce a una mayor acumulación de carbono orgánico en el suelo, que un sistema de producción ganadera basado solo en gramíneas. En estos experimentos, se obtuvieron valores entre 60,6 y 65,1 t.ha ${ }^{-1}$ de $\mathrm{C}$ de carbono acumulado en suelo, en comparación con el sistema tradicional ganadero de pasturas sin árboles, en el cual se detectó una acumulación de 38,3 t.ha ${ }^{-1}$ de C. De igual forma, no se pudo comprobar en estos experimentos una relación entre el grado de mayor o menor complejidad de los arreglos silvopastoriles con mayores o menores cantidades de carbono acumulado en el suelo, pues los tratamientos más complejos evaluados (SSP4), compuesto por el arreglo entre pastura + arbustos forrajeros + árboles forrajeros + árboles maderables, presentaron igual cantidad de carbono acumulado en suelo que en los sistemas más simples (SSP1), compuestos por pastura + arbustos forrajeros.

Los sistemas silvopastoriles mejoran las propiedades físicas del suelo, densidad aparente $(\mathrm{Da})$ y resistencia mecánica del suelo a la penetración de las raíces. Tales efectos se observaron principalmente en la profundidad del suelo superior a $20 \mathrm{~cm}$, atribuidos a la mayor presencia de raíces finas, concomitante con una mayor acumulación de carbono en el suelo, lo cual mejora los niveles de agregación y estructuración de los suelos.

\section{AGRADECIMIENTOS}

Las personas autoras agradecen a la Corporación Colombiana de Investigación Agropecuaria (Agrosavia) y Ministerio de Agricultura y Desarrollo rural (MADR) por el financiamiento de esta investigación, desarrollada dentro del proyecto "Valoración integral de los beneficios de sistemas silvopastoriles de estratos múltiples sobre la sostenibilidad del suelo y la productividad animal en el sistema doble propósito de la región Caribe".

\section{LITERATURA CITADA}

Ayazo, BR; Soto, MR. 2007. Influencia en la estructura vegetal en la comunidad de arañas (Arachnida: Araneae) del suelo en un sistema silvopastoril de Córdoba, Colombia. Tesis Biología. Cordoba, Colombia, Universidad de Córdoba. 113 p.

Barragán, WA; Mahecha, L; Cajas, YS. 2015. Variables fisiológicas-metabólicas de estrés calórico en vacas bajo silvopastoreo y pradera sin árboles. Agronomía Mesoamericana 26(2):211-223.

Burt, R. (Ed.). (2014). Soil Survey Staff. (en línea). In Soil Survey Field and Laboratory Methods ManualSoil Survey Investigations Report. US Department of Agriculture, Natural Resources Conservation Service, USA 51:227-234. Disponible en https:// www.nrcs.usda.gov/Internet/FSE_DOCUMENTS/ stelprdb1244466.pdf

Cáceres, DP. 2012. Cambios en la densidad aparente de los suelos y la materia orgánica y fosforo en suelos del suroeste del Chaco, sometidos a desmonte y siembra de pasturas (en línea). Chaco, Argentina. Consultado ago. 2018. Disponible en https://inta. gob.ar/documentos/cambios-en-la-densidadaparente-materia-organica-y-fosforo-en-suelosdel-suroeste-de-chaco-sometidos-a-desmonte-ysiembra-de-pasturas

Cajas, YS; Sinclair, F. 2001. Characterization of multistrata silvopastoral systems on seasonally dry pastures in the Caribbean Region of Colombia. Agroforestry Systems 53:215-225.

Camargo, JC; Chará, J; Giraldo, L; Chará, A; èPedraza, GX. 2011. Beneficios de los corredores ribereños de Guadua angustifolia en la protección de ambientes acuáticos en la Ecorregión Cafetera de Colombia. 
1. Efectos sobre las propiedades del suelo. Revista Recursos Naturales y Ambiente 61:53-59.

Delgado, R; Castro, L; Cabrera de Bisbal, E; Mujica, M; Caniche, S; Navarro, L; Noguera, I. 2008. Relación entre propiedades físicas del suelo y algunas características del sistema radical del maíz, cultivado en un suelo Fluventic Haplustoll de textura franco-arenosa de Maracay, Venezuela. Agronomía Trop. 58(3):245-255.

Dollinger, J; Shibu, J. 2018. Agroforestry for soil health. Agroforest Syst 92:213-219.

Fahey, TJ; Bledsoe, CS; Day, FP RW; Smucker, AJM. 1999. Chater 20: Fine root production and demography. In Robertson, GP; Coleman, DC; Sollins, P; Bledsoe, CS (eds.). Standard soil methods for long-term ecological research (Vol. 2). Oxford University Press on Demand. p. 437-457.

García, D; Cárdenas, JF; Silva, A. 2018. Evaluación de sistemas de labranza sobre propiedades físicoquímicas y microbiológicas en un Inceptisol. Revista de Ciencias Agrícolas 34(1):16-25.

Holdridge, L. 2000. Ecología basada en zonas de vida. San José, Costa Rica, Instituto Interamericano de Cooperación para la Agricultura, IICA. 225 p.

Ibrahim, M; Chacon, M; Cuartas, CA; Ponce, JF; Vega, P; Casola, F; Rojas, J. 2007. Almacenamiento de carbono en el suelo y la biomasa arbórea en sistemas de usos de tierra en paisajes ganaderos de Colombia, Costa Rica y Nicaragua. Agroforestería en las Américas 45:27-36.

IDEAM (Instituto de Hidrología, Meteorología y Estudios Ambientales); UDCA (Universidad de Ciencias Aplicadas y Ambientales). 2015. Síntesis del estudio nacional de la degradación de suelos por erosión en Colombia 2015. Bogotá D.C., Colombia. IDEAM MADS. 62 p.

ISO (International Organization for Standardization). 2016. Animal feeding stuffs: Determination of moisture and other volatile matter content. 2 ed. Uganda: ISO: 2016, 1- (ISO 6496). 13 p.

Jiménez, RC; Arias, AD. 2004. Distribución de la biomasa y densidad de raíces finas en una gradiente sucesional de bosques en la Zona Norte de Costa Rica. Revista Forestal Mesoamericana Kurú 1(2):44-63.

Lal, R. 2011. Sequestering carbon in soils of agroecosystems. Food Policy 36:S33-S39. Doi: 10.1016/j. foodpol.2010.12.001

Ledesma, LM. 2003. Importancia de los sistemas silvopastoriles y principales limitantes para su implementación en la ganadería colombiana. Revista Colombiana de Ciencias Pecuarias 16(1):11-18.

Linares, AJC. 2006. Evaluación de algunas propiedades del suelo como indicadoras de sostenibilidad para pastoreo rotacional en silvopastoreo en un Endoacuept del valle medio del Rio Sinú Colombia. Trabajo de Grado Magister. Colombia, Convenio
Universidad Nacional de Colombia Sede Palmira, Universidad de Córdoba. $91 \mathrm{p}$.

Martínez, J; Cajas, YS; León, JD; Osorio, NW. 2014. Silvopastoral Systems Enhance Soil Quality in Grasslands of Colombia. Applied and Environmental Soil Science 1-8. Doi: 10.1155/2014/359736

Montagnini, F; Somarriba, E; Murgueitio, E; Fassola, H; Eibl, B; Combe, J; Palma, E. 2015. Sistemas agroforestales: funciones productivas, socioeconómicas y ambientales. Biocenosis (Costa Rica) 2(3):5-6.

Mora, MMA; Ríos, PL; Ríos, RL; Almario, CJ. 2017. Impacto de la actividad ganadera sobre el suelo en Colombia. Ingeniería y Región 17:1-12. Doi: https:// doi.org/10.25054/22161325.1212

Murgueitio, E; Calle, Z; Uribe, F; Calle, A; Solorio, B. 2011. Native trees and shrubs for the productive rehabilitation of tropical cattle ranching lands. Forest Ecol. Manage. 261 (10):1654-1663. Doi: https://doi.org/10.1016/j.foreco.2010.09.027

Ortega, A. 2007. Impacto ambiental de la ganadería de leche en Colombia. In Olivera, M (ed.). Buenas prácticas de producción primaria de leche. Universidad de Antioquia, Facultad de Ciencias Agrarias, Medellín, Colombia. p. 35-43.

Pire, R. 1985. Densidad longitudinal de raíces y extracción de humedad en un viñedo de El Tocuyo, Venezuela. Agronomía Tropical 35(1-3):5-20.

Priano, ME; Fusé, VS; Mestelan, S; Berkovic, AM; Guzmán, SA; Gratton, R; Juliarena, MP. 2017. Afforested sites in a temperate grassland region: influence on soil properties and methane uptake. Agroforestry Systems 92(2):311-320. Doi: https:// doi.org/10.1007/s10457-017-0104-7

Ramachandran, N; Mohan, PK; Kumar, B; Nair, VD. 2009. Agroforestry as a strategy for carbon sequestration. Journal of Plant Nutrition and Soil Science 172(1):10-23.

Rojas, MJ; Ibrahim, M; Ibrahim, M; Andrade, HJ; Andrade, HJ. 2009. Secuestro de carbono y uso de agua en sistemas silvopastoriles con especies maderables nativas en el trópico seco de Costa Rica. Corpoica Cienc. Tecnol. Agropecuaria 10(2):214-223.

Santana, RM; García, PJ; Bustamante, BID; Benavides, BJ; David, HHA. 2012. Biomasa radical de pastos bajo labranza y fertilización en un inceptisol del valle del Sinú. Revista Temas Agrarios 17(2):52-65.

Soil Survey Staff. 2014. Claves para la Taxonomía de Suelos. 14th ed. USDA-Natural Resources Conservation Service, Washington, DC, Estados Unidos de Norte America. 410 p.

Stefano, A; Jacobson, MG. 2017. Soil carbon sequestration in agroforestry systems: a meta-analysis. Agroforest Syst 92(2):285-299. Doi: https://doi.org/10.1007/ s10457-017-0147-9 
Suarez, RSS. 2014. Efecto de los sistemas silvopastoriles sobre el estrés calórico, el comportamiento ingestivo diurno y la ganancia del peso en bovinos romosinuano y brahmán en el valle del Sinú. Tesis Zootecnia. Sincelejo, Sucre, Universidad de Sucre. $52 \mathrm{p}$.

Vallejo, VE; Roldán, F; Dick, RP. 2012. Soil enzymatic activities and microbial biomass in an integrated agroforestry chronosequence compared to monoculture and a native forest in Colombia. Biology and Fertility of Soils 46(6):577-587. Doi: https://doi.org/10.1007/s00374-010-0466-8

Walkley, A; Black, IA. 1934. An examination of the degtjareff method for determining soil organic matter, and a proposed modification of the chromic acid titration method. Soil Science 37(1):29-38. Doi: http://dx.doi.org/10.1097/00010694-19340100000003 